\title{
Children caught in the long shadow of COVID-19
}

\author{
Z Dangor, ${ }^{1} \mathrm{PhD}$; K D Naidoo, ${ }^{2}$ MMed (Paeds); H Saloojee, ${ }^{1} \mathrm{MSc}$; $\mathbf{S}$ A Madhi, ${ }^{3,4} \mathrm{PhD}$ \\ ${ }^{1}$ Department of Paediatrics and Child Health, Faculty of Health Sciences, University of the Witwatersrand, Johannesburg, South Africa \\ ${ }^{2}$ Department of Critical Care, Chris Hani Baragwanath Academic Hospital and Faculty of Health Sciences, University of the Witwatersrand, \\ Johannesburg, South Africa \\ ${ }^{3}$ South African Medical Research Council Vaccines and Infectious Diseases Analytics Research Unit, Faculty of Health Sciences, University of the \\ Witwatersrand, Johannesburg, South Africa \\ ${ }^{4}$ African Leadership in Vaccinology Expertise, Faculty of Health Sciences, University of the Witwatersrand, Johannesburg, South Africa
}

Corresponding author: Z Dangor (ziyaad.dangor@wits.ac.za)

\begin{abstract}
Despite the more transmissible delta variant being associated with higher rates of COVID-19 in unvaccinated adolescents, children have remained relatively spared from severe disease. Nevertheless, children are indirectly affected by the COVID-19 pandemic, which threatens to have far-reaching consequences. The effect of disruptions of seasonal patterns of circulation of respiratory pathogens on future immunity against such pathogens, childhood immunisation programmes, and HIV and tuberculosis treatment programmes poses a threat to the future wellbeing of children. Furthermore, the economic devastation caused by the pandemic, including an increase in unemployment, gives rise to numerous challenges, such as food insecurity, which is likely to worsen childhood nutritional status. Also, COVID-19 has ongoing effects on the mental wellbeing of children, driven in part by the interruption of schooling and other opportunities to socialise. An increase in psychological illnesses has manifested in children consequent to the stresses of the pandemic, lockdowns, caregiver deaths. In this article, we highlight the indirect effects of COVID-19 on children, and suggest solutions to mitigate against the long-term sequelae. A focused health, nutrition, education and child protection response is required from government and healthcare practitioners to safeguard the health and wellbeing of South African children.
\end{abstract}

S Afr J Child Health 2021;15(4):182-184. https://doi.org/10.7196/SAJCH.2021.v15.14.1950

The COVID-19 pandemic, caused by the SARS-CoV-2 virus, represents one of the greatest threats experienced by mankind during the past century, with 245 million reported cases and $>5$ million confirmed deaths by November 2021 worldwide. ${ }^{[1]}$ Notably, the pandemic has been characterised by waves of heightened transmission rates, hospitalisation and death, temporally associated with the easing of movement restrictions, behavioural changes and the emergence of different, more highly transmissible variants.

To date, the major burden of disease has been in the adult population, particularly among individuals $>65$ years of age and in those with underlying medical conditions. Vaccination against SARS-CoV-2 is the most promising and sustainable public intervention to protect against severe COVID-19 and death, but has only been modestly effective in preventing infection and mild disease.

The aspiration to achieve herd immunity against SARS-CoV-2 is receding, even in countries with high vaccine coverage, consequent to the emergence of more transmissible variants of concern, and waning of antibodies associated with declining protection against infection and mild COVID-19. Breakthrough cases in fully vaccinated individuals are, however, less infectious than infections occurring in unvaccinated SARS-CoV-2-naive individuals.

Despite children being as susceptible to infection by SARS-CoV-2 as adults, they have remarkably been spared from developing severe COVID-19 after infection - in contrast to most other infectious diseases. Several reasons have been postulated. Nevertheless, recent trends in high-income, high vaccine-coverage regions have shown a disproportionate rise in COVID-19 infections in unvaccinated adolescents, possibly due to the reduced disease burden in adults because of COVID-19 vaccines being prioritised for them. ${ }^{[2]}$ Another explanation is the emergence of the delta variant, which is twofold more transmissible than the original Wuhan virus. Surveillance data from the National Institute for Communicable Diseases (NICD) show a 1.57 (95\% confidence interval (CI) 1.55 - 1.59) increased odds of disease among children aged 15 - 19 years when comparing the first and third waves. ${ }^{[3]}$ However, the burden of COVID-19 in children is unlikely to ever reach the high rates observed in adults at the peak of the pandemic.

In South Africa (SA), by the end of October 2021, 22 million doses of COVID-19 vaccines had been administered, with 38\% coverage in adults $>18$ years of age. ${ }^{[4]}$ As vaccination rates increase, a return to pre-pandemic behaviour patterns will inevitably result. Despite vaccine coverage rates $>80 \%$ in adults in many high-income countries, the risk of resurgence of COVID-19 persists owing to waning of vaccine-induced antibodies. The risk of resurgence is exacerbated by vaccine hesitancy and lack of access to vaccines in some settings.

Consequently, children remain at ongoing risk of exposure to SARS-CoV-2. Hospitalisation of children with severe COVID-19 and multisystem inflammatory syndrome (MIS-C) has occurred during the previous waves, and children have also been vulnerable to post-acute sequelae of COVID-19 (PASC). Vaccination for 12 - 17-year-old South Africans commenced in October 2021. While the focus of vaccination should primarily be centred on the optimal protection of children with underlying medical conditions, other indirect benefits may include limiting disruptions to schooling, sport and social activities. ${ }^{[5]}$

COVID-19 hospitalisation, MIS-C and PASC may continue to occur in children. However, independent of what transpires next, the long shadow of COVID-19, i.e. its devastating effects on the lives of children currently and over the next decade or longer, is worthy of further consideration. We highlight several other ways in which children have been adversely affected by the pandemic: 
Disproportionately increased burden of other infectious diseases There has been a noticeable decline in the seasonal peaks of common respiratory diseases, such as respiratory syncytial virus (RSV) and influenza, and other common infections. ${ }^{[6]}$ In 2020, Chris Hani Baragwanath Academic Hospital (CHBAH), Johannesburg, SA, child viral surveillance data indicated a virtual disappearance of the RSV peak season ( 25 cases between February and May 2020 compared with 400 - 500 cases in previous years) and no cases of influenza. Consistent with findings in other countries, an increase in RSV cases has been observed outside of the usual seasonal pattern. ${ }^{[7]}$ Nonetheless, concerns have been raised about the possibility of consequent epidemics in the years to come. ${ }^{[8]}$

Many studies have also noted significant reductions in primary healthcare services and child healthcare visits during the COVID-19 pandemic. ${ }^{[9]}$ Importantly, in children, the negative impact on the Expanded Programme on Immunisation (EPI (SA)) and the inadvertent increase in vaccine-preventable diseases are of concern. ${ }^{[9]}$ Although catch-up programmes have been instituted in a number of settings, measures need to be put in place to prevent further erosion of EPI vaccine coverage rates in future COVID-19 waves, particularly in less-resourced settings. ${ }^{[10]}$

\section{Over- and under-nutrition}

The COVID-19 pandemic has shifted dietary and lifestyle habits of many families owing to job losses and disrupted livelihood activities. Many families have been forced to ration food and opt for cheaper and unhealthy food choices. Poor and vulnerable children globally have experienced interruptions in school feeding programmes and access to health services and nutrition-assistance programmes, exacerbating food insecurity. In SA, the National Income Dynamics Study - Coronavirus Rapid Mobile (NIDSCRAM) survey (cramsurvey.org), which followed food insecurity in children through the different waves, concluded in July 2021 that higher household and child hunger rates since the start of the pandemic persisted even after severe lockdown levels were terminated.

In contrast to poorer households, more than a third of members of wealthier families experienced moderate to high weight gains linked to decreased physical activity and fresh food consumption, increased sedentary time, snacking, sweet consumption and eating in response to boredom or anxiety. The poorer diet quality and sedentary behaviours potentially acquired during the prolonged lockdown periods may not be easily reversible in children and their parents.

Modelling studies have predicted dire consequences, particularly acceleration in child wasting, stunting, micronutrient deficiency and overweight rates, with their attendant physical, intellectual, productivity and social impacts. The proposed required responses, not surprisingly, advocate for strengthening of existing strategies and interventions, including identification and management of wasting, support for breastfeeding, maintaining school feeding schemes and social protection measures. ${ }^{[1]]}$

\section{HIV and tuberculosis care}

COVID-19 has ominously impacted on the care of HIV-infected South Africans. During the 2020 lockdown, HIV testing and antiretroviral therapy (ART) initiation decreased by almost half. ${ }^{[12]}$ From a paediatric perspective, not only are testing and access to ART in children essential, but also the negative impact on the prevention of mother-to-child transmission programme could be disastrous missed antenatal visits in HIV-infected pregnant women doubled during COVID-19 lockdown in SA.
Similarly, patients with tuberculosis (TB) have been affected. For the first time in over a decade, the World Health Organization (WHO) reported an increase in TB deaths during the pandemic. ${ }^{[13]}$ Interruptions in diagnostic, therapeutic and follow-up services from the inadvertent redirection of resources to combat the COVID-19 pandemic, as well as reduced healthcare facility visitation by people either afraid of contracting COVID-19 or discouraged by long screening processes and queues, were apparent. The impact of inadequately treated TB increases the possibility of transmission to children in households, creating challenges well beyond the COVID-19 pandemic.

\section{School and education interruption}

Being at school and in a structured environment for learning is important for most children. The benefits of being at school extend beyond the classroom - feeding schemes are important in low-resourced settings. Furthermore, schools are often safe spaces for children at risk of abuse, drugs and mental health problems. ${ }^{[14]}$ Therefore, COVID-19 has widened the already existing educational gap resulting from socioeconomic differences. Moreover, children with learning difficulties will possibly suffer more losses, as most therapy occurs within the school setting. The school interruptions have not only denied children these benefits, but have enormously increased the stress on their caregivers, who are ill-equipped to meet these needs. ${ }^{[15]}$ School interruptions should cease, given that transmission of COVID-19 can be prevented in most education settings, and the evidence that school transmission fuels community transmission is negligible. ${ }^{[16]}$

\section{Loss of caregivers and extended family structures}

With the staggered rollout of the vaccine programme in SA, the relative proportion of younger adults who have died from COVID-19 has increased. Therefore, many more individuals who died during the second and third waves were likely to have been a parent or breadwinner of a family. These deaths have spelled economic ruin for families, with the effects most keenly felt by vulnerable children.

\section{Psychological burden}

Adverse life experiences at a young age are associated with increased risk of mental health disorders in adulthood. Even very young children are not immune to the psychological strain inherent in living during this pandemic. ${ }^{[17]}$ Lockdowns and enforced home confinement have disrupted normal patterns of education, physical activities and socialisation. This situation has had a profound impact on the emotional and social development of young children and adolescents. Manifestations of psychological distress include increased anxiety, clinginess, irritability, inattention, fear and feelings of helplessness. ${ }^{[17]}$ Younger children have also displayed more rebellious behaviour, while teenagers have presented with more affective symptoms. ${ }^{[17]}$ Studies undertaken during earlier phases of the pandemic among adolescents in China and the USA reported an increased incidence of anxiety, depression and post-traumatic stress disorder.

The death of parents and other family members adds heavily to the psychological trauma suffered by children during and even after a possible end to the pandemic. ${ }^{[17]}$ Children from impoverished homes are less likely to access therapy to mitigate the effect of these psychological injuries.

\section{Resource allocation and intensive care services}

Until recently, children suffered a noticeably lower burden of severe COVID-19 than adults. An increase in paediatric COVID-19 and 
other infectious disease burdens threatens to overwhelm existing SA healthcare sector resources. This situation is especially true in the context of critical care, given that only $20 \%$ of intensive care unit (ICU) beds are dedicated to children despite them comprising more than a third of the population.

\section{What response is required?}

The formidable impact of the COVID-19 pandemic on children extends well beyond that of 'usual' viral infections. The indirect effects of COVID-19 on children may have lifelong consequences. An adequate response to the devastation of COVID-19 requires intervention at multiple levels. From a public health perspective, the re-establishment of routine vaccination services for children is a priority. Re-established school nutritional support programmes will partially alleviate hunger in older children, but an accelerated nutrition supplementation support programme for younger children is warranted to manage high hunger and under-nutrition levels.

All schools must remain open, also during future waves, and entire school closure due to local outbreaks must be exceptional and carefully managed. Schools must also play a greater role as sites of psychological support and as areas for the identification and referral of abuse, violence and adolescent pregnancy.

Paediatricians and child health practitioners should be mindful of the psychological burden of disease, reinforce the need for routine childhood immunisations and continue to play a central role in safeguarding the health and wellbeing of children.

The SA government should increase social spending to meet the additional needs of children consequent to the COVID-19 pandemic. These needs include greater investment in health, nutrition, education, child protection and social protection. Although the additional social protection offered through family cash transfers during the lockdown was welcome, ongoing long-term support is required. Appropriate measures could include a substantial increase in the child social support grant and/or the introduction of a basic income grant.

The shadow of COVID-19 on the lives of SA children is indeed long, and getting some sunshine back into the lives of affected children requires a commitment from everyone - be they caregivers or parents, communities, service support staff or government. The future of a generation depends on doing this successfully.

Declaration. None.

Acknowledgements. None.

Author contributions. ZD, KDN, HS and SAM each wrote selected sections. All authors critically appraised and approved the manuscript.
Funding. None.

Conflicts of interest. None.

1. World Health Organization. Coronavirus disease (COVID-19) situation dashboard. 2021. https://www.who.int/ (accessed 29 October 2021).

2. Guilamo-Ramos V, Benzekri A, Thimm-Kaiser M, Hidalgo A, Perlman DC. Reconsidering assumptions of adolescent and young adult severe acute respiratory syndrome Coronavirus 2 transmission dynamics. Clin Infect Dis 2021;73(Suppl 2):S146-S163. https://doi.org/10.1093/cid/ciaa1348

3. National Institute for Communicable Diseases. Epidemiology and clinical characteristics of laboratory confirmed COVID-19 among individuals aged $\leq 19$ years, South Africa, 1 March 2020 - 28 August 2021. https://www.nicd. ac.za/wp-content/uploads/2021/09/COVID-19-in-children-surveillancereport.pdf (accessed 29 October 2021)

4. National Department of Health. Latest vaccine statistics. 2021. https:// sacoronavirus.co.za/latest-vaccine-statistics (accessed 29 October 2021).

5. Anderson EJ, Campbell JD, Creech CB, et al. Warp speed for Coronavirus disease 2019 (COVID-19) vaccines: Why are children stuck in neutral? Clin Infect Dis 2021;73(2):336-340. https://doi.org/10.1093/cid/ciaa1425

6. Yang DD, Ouldali N, Gajdos V, et al. Common pediatric respiratory infectious diseases as possible early predictor for new wave of SARS-CoV-2 infections. Clin Infect Dis 2021;73(2):358-359. https://doi.org/10.1093/cid/ciaa1359

7. Agha R, Avner JR. Delayed seasonal RSV surge observed during the COVID-19 pandemic. Pediatrics 2021;148(3):e2021052089. https://doi.org/10.1542/ peds.2021-052089

8. Di Mattia G, Nenna R, Mancino E, et al. During the COVID-19 pandemic where has respiratory syncytial virus gone? Pediatr Pulmonol 2021;56(10):3106-3109. https://doi.org/10.1002/ppul.25582

9. Jensen C, McKerrow NH. Child health services during a COVID-19 outbreak in KwaZulu-Natal Province, South Africa. S Afr Med J 2020;111(2):114-119. https://doi.org/10.7196/SAMJ.2021.v111i2.15243

10. Ahmed T, Rahman AE, Amole TG, et al. The effect of COVID-19 on maternal newborn and child health (MNCH) services in Bangladesh, Nigeria and South Africa: Call for a contextualised pandemic response in LMICs. Int J Equity Health 2021;20(1):77. https://doi.org/10.1186/s12939-021-01414-5

11. Fore HH, Dongyu Q, Beasley DM, Ghebreyesus TA. Child malnutrition and COVID-19: The time to act is now. Lancet 2020;396(10250):517-518. https:// doi.org/10.1016/s0140-6736(20)31648-2

12. Dorward J, Khubone T, Gate K, et al. The impact of the COVID-19 lockdown on HIV care in 65 South African primary care clinics: An interrupted time series analysis. Lancet HIV 2021;8(3):e158-e165. https://doi.org/10.1016/ S2352-3018(20)30359-3

13. World Health Organization. Global tuberculosis report. 2021. https://www. who.int/publications/i/item/9789240037021 (accessed 29 October 2021).

14. Patrick SW, Henkhaus LE, Zickafoose JS, et al. Well-being of parents and children during the COVID-19 pandemic: A national survey. Pediatrics 2020;146(4):e2020016824. https://doi.org/10.1542/peds.2020-016824

15. Hoofman J, Secord E. The effect of COVID-19 on education. Pediatr Clin North Am 2021;68(5):1071-1079. https://doi.org/10.1016/j.pcl.2021.05.009

16. Kaiser SV, Watson A, Dogan B, et al. Preventing COVID-19 transmission in education settings. Pediatrics 2021;148(3):e2021051438. https://doi. org/10.1542/peds.2021-051438

17. Singh S, Roy D, Sinha K, Parveen S, Sharma G, Joshi G. Impact of COVID-19 and lockdown on mental health of children and adolescents: A narrative review with recommendations. Psychiatr Res 2020;293:113429. https://doi. org/10.1016\%2Fj.psychres.2020.113429

Accepted 5 November 2021 